\title{
Fuzzy Adaptive Control of Uncertain MIMO Chaotic Systems with Unknown Control Direction
}

\author{
Hua Zhang (10 \\ Zhengzhou Preschool Education College, Zhengzhou 450099, China \\ Correspondence should be addressed to Hua Zhang; zhanghua27@163.com
}

Received 5 April 2021; Revised 26 April 2021; Accepted 9 May 2021; Published 19 May 2021

Academic Editor: Shenggang Li

Copyright $\odot 2021$ Hua Zhang. This is an open access article distributed under the Creative Commons Attribution License, which permits unrestricted use, distribution, and reproduction in any medium, provided the original work is properly cited.

This paper presents an adaptive controller for MIMO chaotic systems with system uncertainties and unknown control direction. In the controller design, the matrix decomposition theory is used, and we decompose the control gain matrix into a positive matrix, a diagonal matrix whose diagonal entries are +1 or -1 , and a unity upper triangular matrix. To handle the unknown control direction (i.e., the unknown sign of the control gain matrix), we use the Nussbaum-type function. In addition, we propose an adaptation law named proportional integral (PI) law to update the parameters of the fuzzy system. The stability of the controlled system is proven strictly. Finally, simulation results are presented.

\section{Introduction}

As we all know, most systems are nonlinear and multivariable in the practical control engineering, especially chaotic systems. Chaos is an intrinsic characteristic of nonlinear dynamical systems and a universal phenomenon of nonlinear systems, and there are some vital features such as the sensitive dependency on the initial condition, intrinsic randomness, and irregular order. Chaotic systems can be encountered in many fields, such as mathematics, finance, and physics. According to the characters of dynamic systems, there are usually four classes of chaos systems: temporal chaotic systems, spatiotemporal chaotic systems, spatial chaotic systems, and functional chaotic systems. So, the problem of control chaotic with multi-input and multioutput (MIMO) has received a great interest. Chaotic system control problem was proposed firstly in [1]. After that, an increasing number of methods have been researched and successfully exploited in the control of chaotic system [2], including sliding mode control [3-5], neural adaptive control [6], and fuzzy adaptive control $[7,8]$. Due to the universal approximation theorem [9], the problem of fuzzy adaptive controller designing has aroused an increasing interest. In the last two decades, fuzzy control has a great influence on the control engineering field due to its systematic and efficient frame.
There are two different ways about designing a fuzzy adaptive controller: direct method and indirect method. In the direct method [10], the ideal controller is approximated by the fuzzy system, and the parameters of the fuzzy system are adjusted for achieving control requirements. And in another method [11], the unknown part of the actual system is estimated by the fuzzy system, and then the controller of the system is designed based on these estimated values. By using an indirect method, a problem that calculating the inverse of the control gain matrix is impossible when the matrix is a singular matrix. In order to avoid this problem, an algorithm was proposed in [12], and it is suggested to make the estimated parameters of the fuzzy system inside a compact set where the singularity problem does not exist. However, in [13], it is said that this approach needs a priori knowledge about the set of the parameters and no general approach for obtaining such a set. Another approach about solving this problem is proposed in [14], which uses the regularized inverse matrix of the estimated matrix of the fuzzy system.

Note that a basic assumption is required in the above papers: the sign of the control gain matrix should be known in advance. However, in fact, for most of nonlinear systems, it is very difficult to measure their control direction in practical applications. Therefore, it is very important to solve 
this problem. In order to control the chaotic systems to meet our objective, a function is proposed in [15] named Nussbaum-type function which can be used to solve this problem. Moreover, two assumptions are produced for facilitating the stability analysis of the closed-loop system and the controller designing: a nonlinear system with a lower triangular control structure and the control gain is bounded.

Motivated by the above discussion, in this paper, an adaptive fuzzy controller is implemented for uncertain chaotic systems with unknown control direction. The stability analysis of the closed-loop system is given strictly. Finally, a numerical simulation example is presented for testing the performance. The main contributions of this paper are as follows. (1) Motivated by the theorem of matrix decomposition proposed in [16], the control gain is decomposed into three matrices: a symmetric positive definite matrix, a diagonal matrix with diagonal entries being +1 or -1 , and a unity upper triangular matrix. (2) The Nussbaum-type function [17] is used to estimate the real sign of the gain matrix. (3) A PI adaptation law is proposed to update the fuzzy parameters.

The structure of this work is listed as follows. In Section 2 , the problem description is given. In Section 3, the fuzzy logic system is introduced, and some basic lemmas are also given. In Section 4, the concrete controller design procedure is given, and the stability of the controlled system is proven strictly. Simulation results are included in Section 5. Finally, Section 6 summarizes this work, and future research directions are also discussed.

\section{Problem Description}

A class of nonlinear MIMO chaotic systems is considered as follows:

$$
\dot{X}=A X+f(X)+G(X) u,
$$

where $X=\left[x_{1}, x_{2}, \ldots, x_{n}\right]^{T} \in R^{n}$ is the state vector, $u=\left[u_{1}, \ldots, u_{n}\right]^{T} \in R^{n}$ is the input vector, $A$ is an unknown $n \times n$-dimension matrix, $f(X)=\left[f_{1}(x), \ldots, f_{n}(x)\right] \in R^{n}$, and $G(X)=\left[\begin{array}{ccc}g_{11}(x) & \cdots & g_{1 n}(x) \\ \vdots & \ddots & \vdots \\ g_{n 1}(x) & \cdots & g_{n n}(x)\end{array}\right]$ are unknown continuous nonlinear functions.

Let us denote $X_{d}=\left[x_{d 1}, \ldots, x_{d n}\right]^{T} \in \Omega_{x_{d}} \subset R^{n}$ as the ideal trajectory, where $\Omega_{x_{d}}$ is a known bounded compact set, and the purpose of our work is to design a controller such that the output trajectory can approximate the ideal trajectory $X_{d}$ with a certain precision. Furthermore, all signals involved can remain bounded.

Remark 1. In this paper, system (1) is investigated. However, it should be emphasized that system (1) can represent a large scale of nonlinear systems (chaotic system or not). In addition, most chaotic systems can be expressed by (1). That is, our control method is valid for most existed chaotic systems. One can see that, in the simulation part, a special chaotic system is given for an example.
Then, the tracking error can be defined as

$$
\begin{gathered}
e_{1}=x_{d 1}-x_{1}, \\
\vdots \\
e_{n}=x_{d n}-x_{n} .
\end{gathered}
$$

Let us denote that $E=\left[e_{1}, \ldots, e_{n}\right]^{T}$; then, (2) can be written as

$$
E=X_{d}-X
$$

and $\dot{E}$ can be written as

$$
\dot{E}=\dot{X}_{d}-\dot{X}
$$

Substituting system (1) into (4), we can get

$$
\dot{E}=\dot{X}_{d}-(A X+f(X)+G(X) u) .
$$

Therefore, we can exploit (5) to process the problem of the fuzzy controller design, and then the stability analysis can also be carried out.

\section{Basic Knowledge of the Fuzzy Logic System (FLS)}

A complete FLS includes four parts: fuzzy inference engine, fuzzifier, fuzzy rules, and defuzzifier [18], whose $i$-th fuzzy rule is

$$
R^{(i)} \text { : if } x_{1} \text { is } B_{1}^{i} \text { and ,..., and } x_{n} \text { is } B_{n}^{i} \text {, then } \hat{f} i s f^{i} \text {, }
$$

with $B_{1}^{i}, B_{2}^{i}, \ldots, B_{n}^{i}$ being some fuzzy sets and $f^{i}$ representing the singleton. The FLS is expressed by

$$
\hat{f}(\bar{x})=\frac{\sum_{i=1}^{l} f^{i}\left(\prod_{j=1}^{n} \nu_{B_{j}^{i}}\left(x_{j}\right)\right)}{\sum_{i=1}^{l}\left(\prod_{j=1}^{n} \nu_{B_{j}^{i}}\left(x_{j}\right)\right)=\theta^{T} \psi(\bar{x}),}
$$

with $v_{B^{i}}\left(x_{j}\right)$ being the membership function, and $\theta^{T}=\left[f^{1}, \ldots, f^{l}\right]$ is an adjustable variable, with

$$
\psi^{i}(\bar{x})=\frac{\prod_{j=1}^{n} v_{B_{j}^{i}}\left(x_{j}\right)}{\sum_{i=1}^{l}\left(\prod_{j=1}^{n} v_{B_{j}^{i}}\left(x_{j}\right)\right)} .
$$

\section{Designing a Fuzzy Adaptive Controller and Stability Analysis}

Before starting the main work of this paper, we need to introduce some assumptions and lemmas in advance.Proof. The proof of this lemma can be seen in the literature [19].

Lemma 1. According to Costa et al. [19], it can be concluded that any real matrix $G(X) \in R^{n \times n}$ whose leading principal minors are nonzero can be decomposed into the form as follows:

$$
G(X)=G_{s}(X) D T(X),
$$


where $G_{s}(X) \in R^{n \times n}$ is a symmetric positive definite matrix, $D \in R^{n \times n}$ is a diagonal matrix whose diagonal element is -1 or +1 , and $T(X) \in R^{n \times n}$ is a unity upper triangular matrix.

\section{Assumption 1.}

(1) $G(X)$ is a positive definite or negative definite matrix, and the sign of $G(X)$ is unknown

(2) $G_{s}(X)$ and $(\mathrm{d} / \mathrm{d} t) G_{s}(X)$ are continuous

(3) $\partial g_{i j}(x) / \partial y_{i}^{\left(r_{i}-1\right)}=0, \quad \forall i=1,2, \ldots n \quad$ and $\forall j=1,2, \ldots, n$

\section{Remark 2.}

(1) What we should mention is that the sign of $G(X)$ is unknown in this paper; this means that there are two cases about $G(X)$ : the diagonal element is -1 or +1 .

(2) In order to obtain our control objective, a Nussbaum function is used in the design of the controller, and some important properties of this function are listed as follows:

(a) $\lim _{s \longrightarrow \infty} \sup (1 / s) \int_{0}^{s} N(\zeta) \mathrm{d} \zeta=+\infty$

(b) $\lim _{s \longrightarrow \infty} \inf (1 / s) \int_{0}^{s} N(\zeta) \mathrm{d} \zeta=-\infty$

(3) There are some examples about the Nussbaum function from the literature:

$$
\begin{aligned}
& N_{1}(\zeta)=\zeta^{2} \cos (\zeta) \\
& N_{2}(\zeta)=\zeta \cos (\sqrt{|\zeta|}) \\
& N_{3}(\zeta)=\cos \left(\frac{\pi}{2} \zeta\right) e^{\zeta^{2}}
\end{aligned}
$$

and in this paper, we will use the function $N_{3}(\zeta)$, and it is easy to know that $\dot{\zeta}=\sum_{i=1}^{n}\left[\theta_{i}^{T} \psi_{i}(z)\right]$.

Now, we will introduce another lemma about the Nussbaum function that will be used in the following stability analysis.Proof. The proof of this lemma is given in $\mathrm{Ge}$ and Jing Wang [20].

Lemma 2. $V(t)$ and $\zeta(t)$ are of $C^{1}$ whose definition domain is $\left[0, t_{f}\right)$, with $V(t) \geq 0 \forall \in\left[0, t_{f}\right)$. Let $N(t)$ be a Nussbaum function; then,

$$
V(t) \leq c_{0} \pm \int_{0}^{t}(g N(\zeta) \pm 1) \dot{\zeta} \mathrm{d} \tau
$$

with $g>0, \quad c_{0}$ being a suitable constant, and $\int_{0}^{t}(g N(\zeta) \pm 1) \dot{\zeta} d \tau$ being bounded on $\left[0, t_{f}\right)$.

Because matrix $A$ is unknown, the function $f(x)$ and the sign of $G(X)$ are not known. We need to use FLSs to design our controllers.

Remark 3. As we all know, there are two methods about designing a fuzzy adaptive control system: the direct method and the indirect method. The most important difference between two methods is the control object: the direct method is using the fuzzy system to estimate the ideal controller, and the indirect method is using the fuzzy system to estimate the unknown part of the system such as matrix $A$, the function $f(x)$, and the sign of $G(X)$. In this paper, we will exploit the indirect method to design the fuzzy controller that we need.

By using Lemma 1, matrix $G(X)$ can be decomposed into $G_{s}(X) D T(X)$. Substituting it into system (5), we can get

$$
\begin{aligned}
G_{1}(X) \dot{E}= & G_{1}(X)\left[\dot{X}_{d}-(A X+f(X))\right] \\
& -(D T(X)-D)-D u \\
= & F(X, u)-D u,
\end{aligned}
$$

where $G_{1}(X)=G_{s}^{-1}(X)$ and $F(X, u)=G_{1}(X)\left[\dot{X}_{d}-(A X+\right.$ $f(X))]-(D T(X)-D)$. Let us denote $\alpha(z)=(1 / 2) \dot{G}_{1}(X)$ $E+F(X, u)$; then, (11) can be transformed into the following form:

$$
G_{1}(X) \dot{E}=-\frac{1}{2} \dot{G}_{1}(X) E+\alpha(z)-D u
$$

Remark 4. By checking the expressing form of $\alpha(z)$ and $F(X, u)$, we can find that the structure of vector $z$ is an upper triangular control structure. The element of vector $z$ can be selected by

$$
\begin{aligned}
z_{1} & =\left[X, E, u_{1}, \ldots, u_{n}\right], \\
z_{2} & =\left[X, E, u_{2}, \ldots, u_{n}\right], \\
& \vdots \\
z_{n-1} & =\left[X, E, u_{n-1}, u_{n}\right], \\
z_{n} & =\left[X, E, u_{n}\right] .
\end{aligned}
$$

Define the compact sets as follows:

$$
D_{z_{i}}=\left[X, E, u_{i}, \ldots, u_{n}\right], \quad i=1, \ldots, n
$$

Remark 5. Noting that $E$ and $u_{i}$ are functions of vector $X$ and $X_{d}$ is bounded, we can know that $z_{i}=X$.

Now, we will use the fuzzy system to approximate $\alpha(z)$. We can use the FLS as follows:

$$
\widehat{\alpha}_{i}\left(z_{i}, \theta_{i}\right)=\theta_{i}^{T} \psi_{i}\left(z_{i}\right) .
$$

Let us denote the ideal parameters of $\theta_{i}$ as

$$
\theta_{i}^{*}=\arg \min _{\theta_{i}}=\left[\sup _{z_{i} \in D_{z_{i}}}\left|\alpha_{i}\left(z_{i}\right)-\widehat{\alpha}_{i}\left(z_{i}, \theta_{i}\right)\right|\right] \text {. }
$$

We should notice that the ideal parameters of $\theta_{i}$ are just introduced for analysis, and we do not need to know their true value. 
Let us define

$$
\tilde{\theta}_{i}=\theta_{i}-\theta_{i}^{*}
$$

as the error of the parameter estimation; then, we have

$$
\varepsilon_{i}\left(z_{i}\right)=\alpha_{i}\left(z_{i}\right)-\widehat{\alpha}_{i}\left(z_{i}, \theta_{i}^{*}\right) .
$$

According to Chang [21], we can assume that our fuzzy system is not contrary to the universal approximate principle on the compact set $D_{z}$, and we assume this set is big enough. Then, our input vector of the fuzzy system can remain within $D_{z}$ under the closed-loop system. In summary, we can assume that the error of the parameter estimation is bounded on the compact set $D_{z}$, i.e., for any $z \in D_{z}$,

$$
\left|\varepsilon_{i}\left(z_{i}\right)\right| \leq \varepsilon_{i},
$$

with $\varepsilon_{i}$ being an arbitrarily small positive constant.

Then, let us denote

$$
\begin{aligned}
\widehat{\alpha}(z, \theta) & =\theta^{T} \psi(z)=\left[\widehat{\alpha_{1}}\left(z_{1}, \theta_{1}\right), \ldots, \widehat{\alpha_{n}}\left(z_{n}, \theta_{n}\right)\right], \\
\mathcal{E}(z) & =\left[\varepsilon_{1}\left(z_{1}\right), \ldots, \varepsilon_{n}\left(z_{n}\right)\right], \\
\mathcal{E} & =\left[\varepsilon_{1}, \ldots, \varepsilon_{n}\right] .
\end{aligned}
$$

Then, we have

$$
\begin{aligned}
\widehat{\alpha}(z, \theta)-\alpha(z) & =\widehat{\alpha}(z, \theta)-\widehat{\alpha}\left(z, \theta^{*}\right)+\widehat{\alpha}\left(z, \theta^{*}\right)-\alpha(z) \\
& =\widehat{\alpha}(z, \theta)-\widehat{\alpha}\left(z, \theta^{*}\right)-\varepsilon(z) \\
& =\tilde{\theta} \psi(z)-\varepsilon(z),
\end{aligned}
$$

with $\tilde{\theta} \psi(z)=\left[\tilde{\theta_{1}} \psi\left(z_{1}\right), \ldots, \tilde{\theta_{n}} \psi\left(z_{n}\right)\right]$. Now, in order to satisfy our control purpose, the controller can be designed in the following form:

$$
u=N(\zeta)\left[\theta^{T} \psi(z)+K_{1} E+K_{0} \operatorname{Sign}(E)\right]
$$

with

$$
\begin{aligned}
& K_{0}=\operatorname{diag}\left[k_{01}, k_{02}, \ldots, k_{0 n}\right], \\
& K_{1}=\operatorname{diag}\left[k_{11}, k_{12}, \ldots, k_{1 n}\right],
\end{aligned}
$$

and $k_{0 i}>0, i=1, \ldots, n$, and $k_{1 i}>0, i=1, \ldots, n$.

To update parameter $\theta_{i}$, we can design a PI adaptation law:

$$
\theta_{i}=\int_{0}^{t}\left[-\sigma_{i} \gamma_{0 i}\left|e_{i}\right| \theta_{i}+\gamma_{0 i} e_{i} \psi_{i}\left(z_{i}\right)\right] \mathrm{d} \tau-\gamma_{1 i} \delta_{i},
$$

with $\delta_{i}=\sigma_{i}\left|e_{i}\right| \theta_{i}-e_{i} \psi_{i}\left(z_{i}\right)$ and $\gamma_{0 i}, \gamma_{1 i}, \sigma_{i}>0$ being constants.Proof. Let us denote

$$
\begin{aligned}
& V_{1}=\frac{1}{2} E^{T} G_{1}(X) E, \\
& V_{2}=\sum_{i=1}^{n} \frac{1}{2 \gamma_{0 i}}\left(\widetilde{\theta}_{i}+\gamma_{1 i} \delta_{i}\right)^{T}\left(\tilde{\theta}_{i}+\gamma_{1 i} \delta_{i}\right) .
\end{aligned}
$$

Remark 6. Note that there are some related fuzzy control methods which have been proposed recently, for example, in [22-24]. However, the proposed method is different from the above literature because a PI law is designed in this paper. We should mention the difference between the two terms in PI law (24), $\sigma_{i} \gamma_{0 i}\left|e_{i}\right| \theta_{i}$ and $\delta_{i}$. The purpose of the term $\sigma_{i} \gamma_{0 i}\left|e_{i}\right| \theta_{i}$ is to ensure that parameter $\theta_{i}$ is bounded, and another one is a proportional term, which can ensure that parameter $\theta_{i}$ has a fast convergence.

Theorem 1. Consider system (5). Using controller (22) with PL law (24), the following properties can be ensured:

(1) All signals involved are bounded

(2) The tracking errors are asymptotically stable

Then, we consider the following Lyapunov function:

$$
V=V_{1}+V_{2}
$$

First, through the above analysis, we can get

$$
\begin{aligned}
G_{1}(X) \dot{E} & =-\frac{1}{2} \dot{G}_{1}(X) E+\alpha(z)-D u \\
& =-\frac{1}{2} \dot{G}_{1}(X) E-K_{1} E-K_{0} \operatorname{Sign}(E)-\tilde{\theta} \psi(z)+\varepsilon(z)+\left[\theta^{T} \psi(z)+K_{1} E+K_{0} \operatorname{Sign}(E)\right]-D u
\end{aligned}
$$

Substituting controller (22) into (27), we have

$$
G_{1}(X) \dot{E}=-\frac{1}{2} \dot{G}_{1}(X) E-K_{1} E-K_{0} \operatorname{Sign}(E)-\tilde{\theta} \psi(z)+\varepsilon(z)+\left[\theta^{T} \psi(z)+K_{1} E+K_{0} \operatorname{Sign}(E)\right][1+g N(\zeta)]
$$

with $g=d_{i i}, \forall i=1, \ldots, n$. 

have

Multiplying $E^{T}$ to both sides of the above equation, we

$$
\begin{aligned}
E^{T} G_{1}(X) \dot{E}= & -\frac{1}{2} E^{T} \dot{G}_{1}(X) E-\sum_{i=1}^{n} k_{1 i} e_{i}^{2}-\sum_{i=1}^{n} k_{0 i}\left|e_{i}\right|-\sum_{i=1}^{n} e_{i} \widetilde{\theta}_{i}^{T} \psi_{i}\left(z_{i}\right)+\sum_{i=1}^{n} e_{i} \varepsilon_{i}\left(z_{i}\right) \\
& +\sum_{i=1}^{n} e_{i}\left[\theta_{i}^{T} \psi_{i}\left(z_{i}\right)+k_{1 i} e_{i}+k_{0 i} \operatorname{sign}\left(e_{i}\right)\right][1+g N(\zeta)] .
\end{aligned}
$$

Then, we have

$$
\begin{aligned}
\dot{V}_{1}= & \frac{1}{2} E^{T} \dot{G}_{1}(X) E+E^{T} G_{1}(X) \dot{E} \\
= & -\sum_{i=1}^{n} k_{1 i} e_{i}^{2}-\sum_{i=1}^{n} k_{0 i}\left|e_{i}\right|-\sum_{i=1}^{n} e_{i} \widetilde{\theta}_{i}^{T} \psi_{i}\left(z_{i}\right)+\sum_{i=1}^{n} e_{i} \varepsilon_{i}\left(z_{i}\right) \\
& +\sum_{i=1}^{n} e_{i}\left[\theta_{i}^{T} \psi_{i}\left(z_{i}\right)+k_{1 i} e_{i}+k_{0 i} \operatorname{sign}\left(e_{i}\right)\right][1+g N(\zeta)] \\
= & -\sum_{i=1}^{n} k_{1 i} e_{i}^{2}-\sum_{i=1}^{n} k_{0 i}\left|e_{i}\right|-\sum_{i=1}^{n} e_{i} \widetilde{\theta}_{i}^{T} \psi_{i}\left(z_{i}\right)+\sum_{i=1}^{n} e_{i} \varepsilon_{i}\left(z_{i}\right)+[1+g N(\zeta)] \dot{\zeta} .
\end{aligned}
$$

Next, let us analyze $V_{2}$ as

$$
\begin{aligned}
\dot{V}_{2} & =\sum_{i=1}^{n} \frac{1}{\gamma_{0 i}}\left(\dot{\tilde{\theta}}_{i}+\gamma_{1 i} \dot{\delta}_{i}\right)^{T}+\left(\widetilde{\theta}_{i}+\gamma_{1 i} \delta_{i}\right) \\
& =\sum_{i=1}^{n} \frac{1}{\gamma_{0 i}}\left(-\sigma_{i} \gamma_{0 i}\left|e_{i}\right| \theta_{i}+\gamma_{0 i} e_{i} \psi_{i}\left(z_{i}\right)\right)^{T}\left(\widetilde{\theta}_{i}+\gamma_{1 i} \delta_{i}\right) \\
& =\sum_{i=1}^{n}-\delta_{i}^{T}\left(\tilde{\theta}_{i}+\gamma_{1 i} \delta_{i}\right) \\
& =\sum_{i=1}^{n}\left(\tilde{\theta}_{i}^{T}\left(-\sigma_{i}\left|e_{i}\right| \theta_{i}+e_{i} \psi_{i}\left(z_{i}\right)\right)-\gamma_{1 i}\left\|\delta_{i}\right\|^{2}\right) \\
& =\sum_{i=1}^{n}\left(-\sigma_{i}\left|e_{i}\right| \widetilde{\theta}_{i}^{T} \theta_{i}-\gamma_{1 i}\left\|\delta_{i}\right\|^{2}+e_{i} \widetilde{\theta}_{i}^{T} \psi_{i}\left(z_{i}\right)\right) \\
& \leq \sum_{i=1}^{n}\left(\sigma_{i}\left|e_{i}\right|\left(-\frac{1}{2}\left\|\widetilde{\theta}_{i}\right\|^{2}+\frac{1}{2}\left\|\theta_{i}^{*}\right\|^{2}\right)+e_{i} \widetilde{\theta}_{i}^{T} \psi_{i}\left(z_{i}\right)-\gamma_{1 i}\left\|\delta_{i}\right\|^{2}\right) .
\end{aligned}
$$

From (30) and (31), we have 


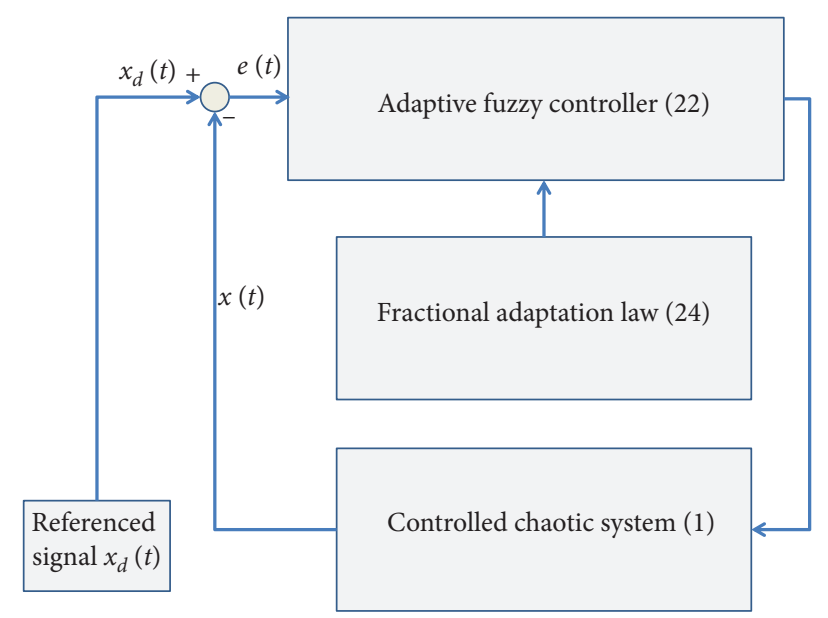

FIGURE 1: Block diagram of this work.

$$
\begin{aligned}
\dot{V} & =\dot{V}_{1}+\dot{V}_{2} \\
& \leq-\sum_{i=1}^{n} k_{1 i} e_{i}^{2}-\sum_{i=1}^{n} k_{0 i}\left|e_{i}\right|+\sum_{i=1}^{n} e_{i} \varepsilon_{i}\left(z_{i}\right)-\frac{1}{2} \sum_{i=1}^{n} \sigma_{i}\left|e_{i}\right|\left\|\widetilde{\theta}_{i}\right\|^{2}+\frac{1}{2} \sum_{i=1}^{n} \sigma_{i}\left|e_{i}\right|\left\|\theta_{i}^{*}\right\|^{2}+[1+g N(\zeta)] \dot{\zeta} \\
& \leq-\sum_{i=1}^{n} k_{1 i} e_{i}^{2}-\sum_{i=1}^{n} k_{0 i}\left|e_{i}\right|+\sum_{i=1}^{n}\left|e_{i}\right| \varepsilon_{i}+\frac{1}{2} \sum_{i=1}^{n} \sigma_{i}\left|e_{i}\right|\left\|\theta_{i}^{*}\right\|^{2}+[1+g N(\zeta)] \dot{\zeta}
\end{aligned}
$$

If we choose $k_{0 i} \geq \varepsilon_{i}+(1 / 2) \sigma_{i}\left\|\theta_{i}^{*}\right\|^{2}$, then we can get

$$
\dot{V} \leq-\sum_{i=1}^{n} k_{1 i} e_{i}^{2}+[1+g N(\zeta)] \dot{\zeta} .
$$

By using Lemma 2, integrating the above integral over $[0, t]$, we have

$$
\begin{aligned}
& V(t) \leq V(t)+\int_{0}^{t} \sum_{i=1}^{n} k_{1 i} e_{i}^{2} \mathrm{~d} \tau \\
& \leq V(0)+\int_{0}^{t}(g N(\zeta) \pm 1) \dot{\zeta} \mathrm{d} \tau .
\end{aligned}
$$

According to Lemma 2, we can know that $V(t), \zeta(t)$, and $\int_{0}^{t}(g N(\zeta) \pm 1) \dot{\zeta} \mathrm{d} \tau$ are bounded on $\left(0, t_{f}\right)$. When $t_{f} \longrightarrow \infty$, the conclusion is also true (the proof can be seen in Xu et al. [25]). So, $e_{i}$ is bounded. From (34), we can conclude that $\int_{0}^{t} \sum_{i=1}^{n} k_{1 i} e_{i}^{2} \mathrm{~d} \tau$ is bounded, i.e., $e_{i} \in L_{2}$. In (27), all signals involved on the right side are bounded, and note that the functions $G_{1}(X)$ and $\dot{G}(x)$ are continuous functions, so it can be easy to show that $\dot{e}_{i} \in L_{\infty}$. Finally, we can conclude that $e_{i} \longrightarrow 0$ as $t \longrightarrow \infty$ by using the Barbalat lemma (see Slotine et al. [26]).

Remark 7. There is still another choice of $\dot{\zeta}$ and controller $u$. In Yao and Pan [27], Table 1 summarizes this choice, and the proof is similar to the proof of Theorem 1 . This means all choices of Table 1 in [27] can guarantee that all signals involved in this closed-loop system are bounded, and the errors converge to zero.

Remark 8. (1) For eliminating the chattering effect caused by robust term Sign $\left(e_{i}\right)$ of the controller, we can use some smooth functions to replace it, such as tanh or arctan. (2) Because the value of $\theta_{i}^{*}$ is unknown, the choice of parameters $k_{0 i}$ is difficult. However, we can use an adaptation law $\dot{k}_{0 i}=$ $\gamma_{2 i}\left|e_{i}\right|$ to replace it, where $\gamma_{2 i}>0$.

Overall, the framework of this paper is shown in Figure 1.

\section{Numerical Simulation}

In this section, the effectiveness of the controller that we designed in this paper is shown. A chaotic financial system is used for simulation.

The MIMO chaotic financial system is given by

$$
\begin{aligned}
& \dot{x}_{1}=x_{3}+\left(x_{2}-a\right) x_{1}+\left(1+x_{1}^{2}\right) u_{1}(t), \\
& \dot{x}_{2}=1-b x_{2}-x_{1}^{2}+\left(1+x_{2}^{2}\right) u_{2}(t), \\
& \dot{x_{3}}=-x_{1}-c x_{3}+\left(1+x_{3}^{2}\right) u_{3}(t)
\end{aligned}
$$



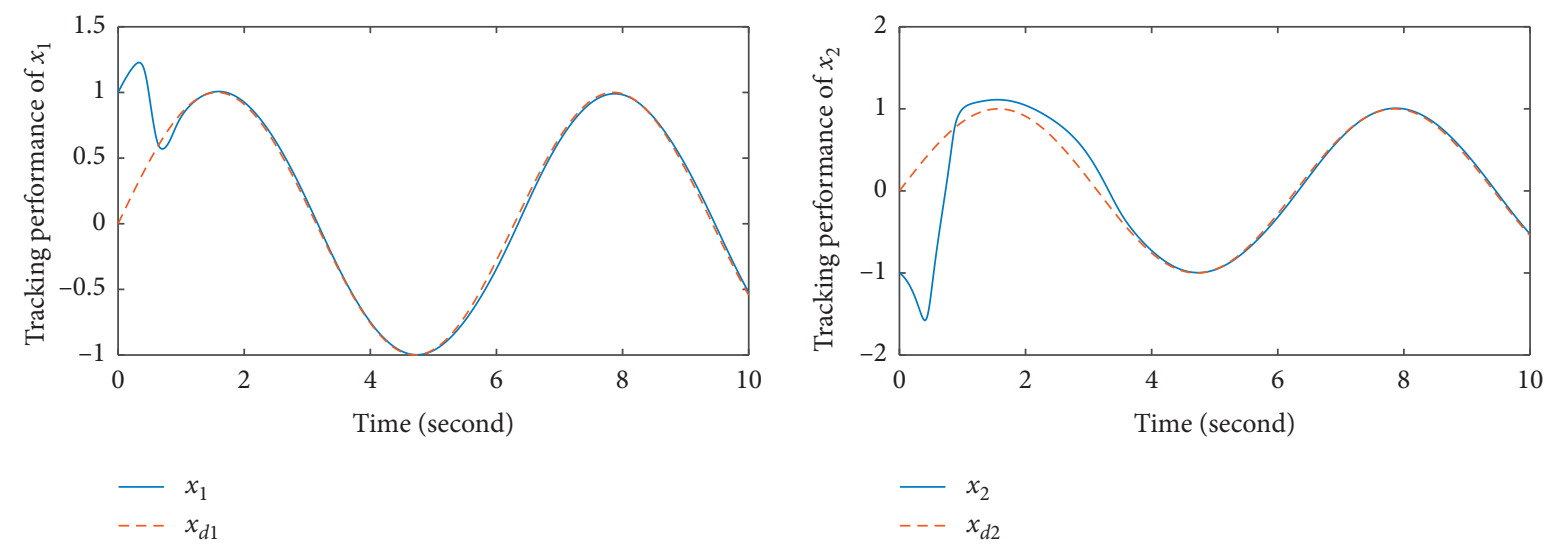

(a)
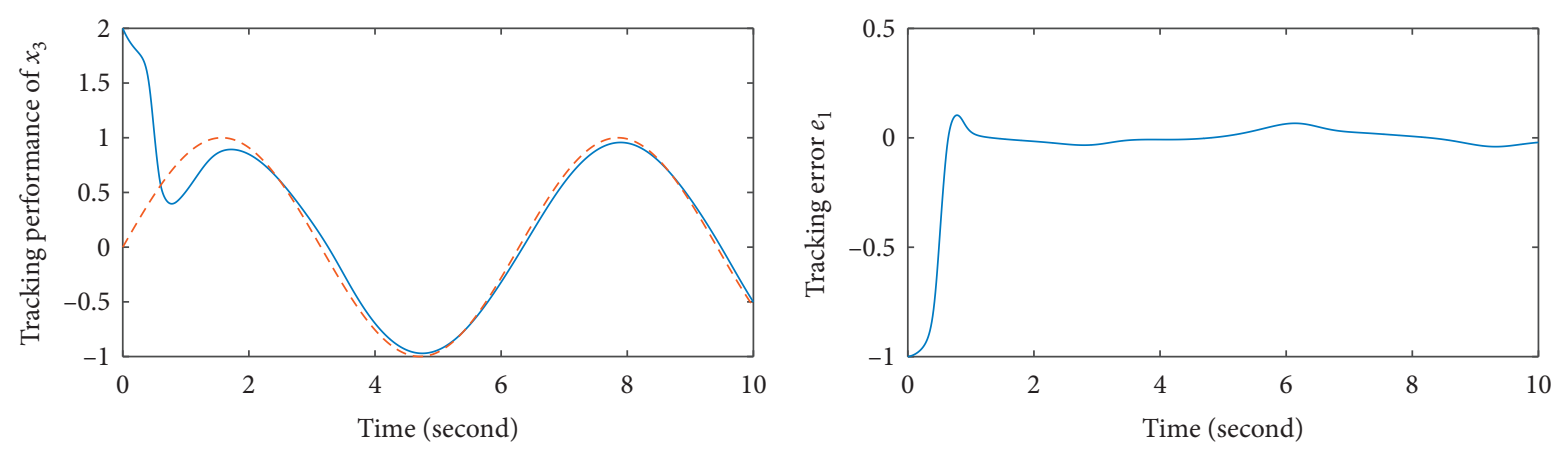

$-x_{3}$

(c)

(d)

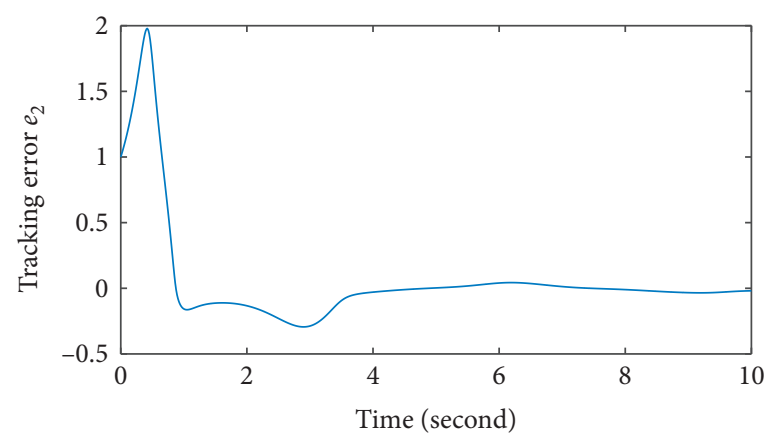

(e)

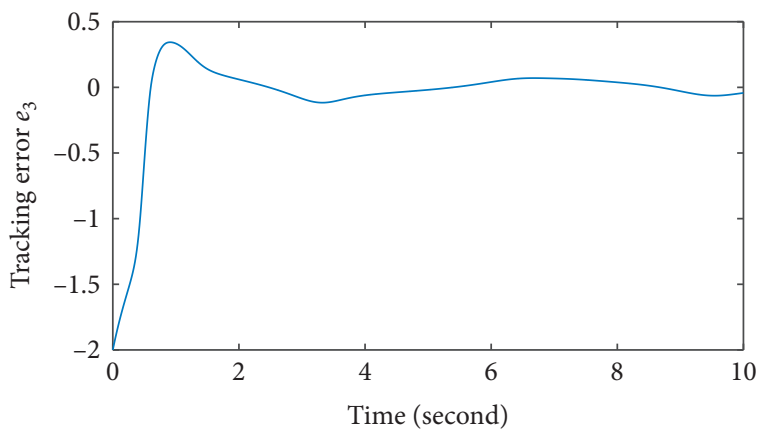

(f)

FIGURE 2: Simulation results for $x_{i}(t)$ and $e_{i}(t)$ in (a) tracking of $x_{d 1}$, (b) tracking of $x_{d 2}$, (c) tracking of $x_{d 3}$, (d) time response of $e_{1}$, (e) time response of $e_{2}$, and (f) time response of $e_{3}$.

where $a, b$, and $c$ are system parameters. Let us denote $X=$ $\left[x_{1}, x_{2}, x_{3}\right]^{T}$ and $u=\left[u_{1}, u_{2}, u_{3}\right]^{T}$; then, system (35) can be expressed as follows:

$$
\dot{X}=A X+f(X)+G(X) u,
$$

where 


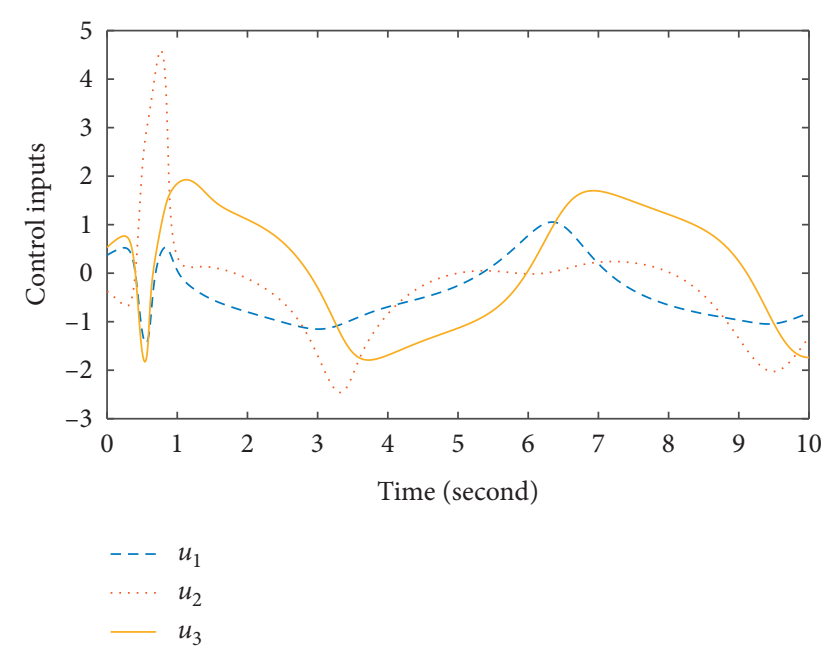

Figure 3: Simulation results for $u_{1}(t), u_{2}(t)$, and $u_{3}(t)$.

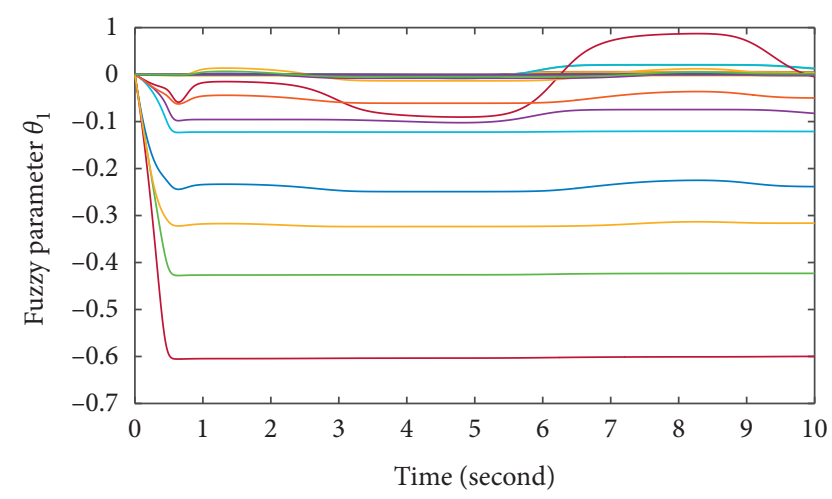

FIGURE 4: Simulation results for $\theta_{1}$.

$$
\begin{aligned}
A & =\left[\begin{array}{ccc}
-a & 0 & 1 \\
0 & -b & 0 \\
-1 & 0 & -c
\end{array}\right], \\
f(X) & =\left[\begin{array}{l}
f_{1}(X) \\
f_{2}(X) \\
f_{3}(X)
\end{array}\right]=\left[\begin{array}{c}
x_{1} x_{2} \\
-x_{1}^{2}+1 \\
0
\end{array}\right], \\
G(X) & =\left[\begin{array}{ccc}
1+x_{1}^{2} & 0 & 0 \\
0 & 1+x_{2}^{2} & 0 \\
0 & 0 & 1+x_{3}^{2}
\end{array}\right] .
\end{aligned}
$$

The ideal trajectories are $x_{d 1}=\sin (t), x_{d 2}=\sin (t)$, and $x_{d 3}=\sin (t)$, and the initial conditions are $X(0)=[1,-1,2]$, $\theta_{1}=0, \theta_{2}=0, \theta_{3}=0$, and $\zeta(0)=0$.

In the simulation example, we use three fuzzy systems. The input of each system is the state variable of the system. For each input (that is, each system state variable), we define four fuzzy membership functions in the interval $[-7,7]$. The design parameters in this simulation are selected as follows: $a=0.78, b=0.2, c=1.5, k_{1 i}=0.5(i=1,2,3), k_{1 i}=0.5(i=$ $1,2,3), \gamma_{0 i}=200(i=1,2,3)$, and $\sigma_{i}=0.02(i=1,2,3)$.

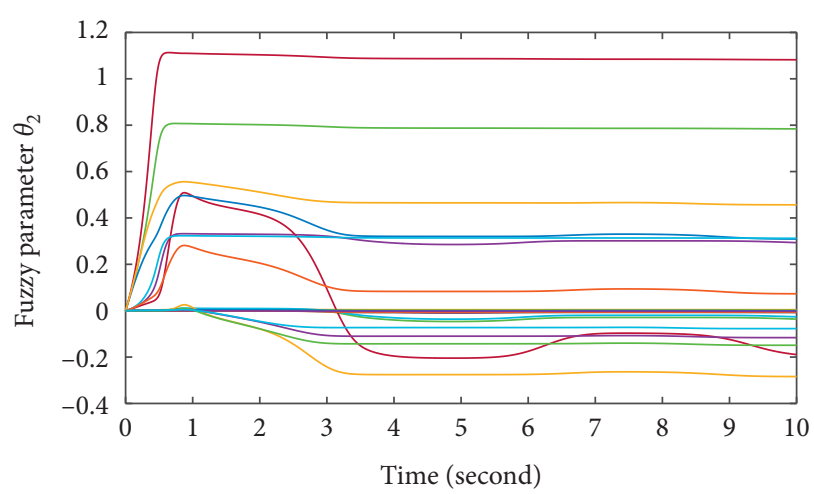

FIgURE 5: Simulation results for $\theta_{2}$.

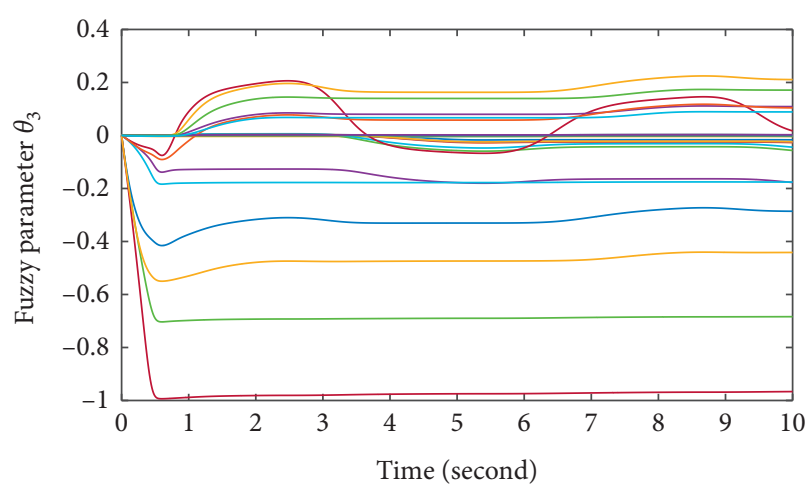

FIgURE 6: Simulation results for $\theta_{3}$.

The simulation results are shown in Figures 2-6. Figure 2 shows the trajectory of $x_{i}, i=1,2,3$, and the tracking error $e_{i}, i=1,2,3$. One can see that the performance is very good. Figure 3 shows the controller $u_{i}(t), i=1,2,3$. In Figures 4-6, the results of parameters $\theta_{1}, \theta_{2}$, and $\theta_{3}$, respectively, are presented.

\section{Conclusions}

In this study, a novel fuzzy adaptive controller for the MIMO chaotic system with unknown control direction has been proposed. A type of function called Nussbaum function has been used in the designing of the controller. We also proposed a PI law to update the fuzzy parameters. Finally, in the simulation results, we can see that all signals involved in this closed system are bounded, and the tracking errors all converge to zero as $t \longrightarrow \infty$.

\section{Data Availability}

All the datasets generated for this study are included within this manuscript.

\section{Conflicts of Interest}

The author declares that there are no conflicts of interest regarding the publication of this paper. 


\section{References}

[1] C. Grebogi, E. Ott, and J. A. Yorke, "Crises, sudden changes in chaotic attractors, and transient chaos," Physica D: Nonlinear Phenomena, vol. 7, no. 1-3, pp. 181-200, 1983.

[2] G. Chen and X. Dong, "From chaos to order-perspectives and methodologies in controlling chaotic nonlinear dynamical systems," International Journal of Bifurcation and Chaos, vol. 3, no. 6, pp. 1363-1409, 1993.

[3] G. Ablay, "Sliding mode control of uncertain unified chaotic systems," Nonlinear Analysis: Hybrid Systems, vol. 3, no. 4, pp. 531-535, 2009.

[4] H. Liu, H. Wang, J. Cao, A. Alsaedi, and T. Hayat, "Composite learning adaptive sliding mode control of fractional-order nonlinear systems with actuator faults," Journal of the Franklin Institute, vol. 356, no. 16, 2019.

[5] S. Ha, H. Liu, S. Li, and A. Liu, "Backstepping-based adaptive fuzzy synchronization control for a class of fractional-order chaotic systems with input saturation," International Journal of Fuzzy Systems, vol. 21, no. 5, pp. 1571-1584, 2019.

[6] I. Abadlia, L. Hassaine, A. Beddar, F. Abdoune, and M. R. Bengourina, "Adaptive fuzzy control with an optimization by using genetic algorithms for grid connected a hybrid photovoltaic-hydrogen generation system," International Journal of Hydrogen Energy, vol. 45, no. 43, pp. 22589-22599, 2020.

[7] A. Boulkroune, A. Bouzeriba, S. Hamel, and T. Bouden, "Adaptive fuzzy control-based projective synchronization of uncertain nonaffine chaotic systems," Complexity, vol. 21, no. 2, pp. 180-192, 2015.

[8] T. Zhou, C. Liu, X. Liu, H. Wang, and Y. Zhou, "Finite-time prescribed performance adaptive fuzzy control for unknown nonlinear systems," Fuzzy Sets and Systems, vol. 402, pp. 16-34, 2021.

[9] A. Boulkroune, M. Tadjine, M. M'Saad, and M. Farza, “A unified approach for design of indirect adaptive outputfeedback fuzzy controller," International Journal of Intelligent Systems Technologies and Applications, vol. 5, no. 1-2, pp. 83-103, 2008.

[10] Y.-C. Chang, "Robust tracking control for nonlinear mimo systems via fuzzy approaches," Automatica, vol. 36, no. 10, pp. 1535-1545, 2000.

[11] N. Essounbouli, A. Hamzaoui, and J. Zaytoon, "An improved robust adaptive fuzzy controller for mimo systems," Control and Intelligent Systems, vol. 34, no. 1, pp. 12-21, 2006.

[12] J. T. Spooner and K. M. Passino, "Stable adaptive control using fuzzy systems and neural networks," IEEE Transactions on Fuzzy Systems, vol. 4, no. 3, pp. 339-359, 1996.

[13] L.-X. Wang, "Stable adaptive fuzzy control of nonlinear systems," IEEE Transactions on Fuzzy Systems, vol. 1, no. 2, pp. 146-155, 1993.

[14] S. Tong and Y. Li, "Adaptive fuzzy output feedback control for switched nonlinear systems with unmodeled dynamics," IEEE Transactions on Cybernetics, vol. 47, no. 2, pp. 295-305, 2017.

[15] R. D. Nussbaum, "Some remarks on a conjecture in parameter adaptive control," Systems \& Control Letters, vol. 3, no. 5, pp. 243-246, 1983.

[16] N. Hovakimyan, F. Nardi, A. Calise, and N. Nakwan Kim, "Adaptive output feedback control of uncertain nonlinear systems using single-hidden-layer neural networks," IEEE Transactions on Neural Networks, vol. 13, no. 6, pp.1420-1431, 2002.

[17] B. Ren, S. S. Ge, K. P. Tee, and T. H. Lee, "Adaptive neural control for output feedback nonlinear systems using a barrier
Lyapunov function," IEEE Transactions on Neural Networks, vol. 21, no. 8, pp. 1339-1345, 2010.

[18] S. Tong and H.-X. Li, "Fuzzy adaptive sliding-mode control for mimo nonlinear systems," IEEE Transactions on Fuzzy Systems, vol. 11, no. 3, pp. 354-360, 2003.

[19] R. R. Costa, H. Liu, A. K. Imai, and P. Kokotovi, "Lyapunovbased adaptive control of mimo systems," Automatica, vol. 39, no. 7, pp. 1251-1257, 2003.

[20] S. S. Ge and W. Jing Wang, "Robust adaptive neural control for a class of perturbed strict feedback nonlinear systems," IEEE Transactions on Neural Networks, vol. 13, no. 6, pp. 1409-1419, 2002.

[21] Y.-C. Chang, "Adaptive fuzzy-based tracking control for nonlinear SISO systems via VSS and h/sup/spl infin//approaches," IEEE Transactions on Fuzzy Systems, vol. 9, no. 2, pp. 278-292, 2001.

[22] M. Chen, H. Wang, and X. Liu, "Adaptive fuzzy practical fixed-time tracking control of nonlinear systems," IEEE Transactions on Fuzzy Systems, vol. 29, no. 3, pp. 664-673, 2019.

[23] W. He and Y. Dong, "Adaptive fuzzy neural network control for a constrained robot using impedance learning," IEEE Transactions on Neural Networks and Learning Systems, vol. 29, no. 4, pp. 1174-1186, 2017.

[24] H. Wang, W. Bai, X. Zhao, and P. X. Liu, "Finite-time-prescribed performance-based adaptive fuzzy control for strict-feedback nonlinear systems with dynamic uncertainty and actuator faults," IEEE Transactions on Cybernetics, pp. 1-13, 2021.

[25] B. Xu, X. Wang, and Z. Shi, "Robust adaptive neural control of nonminimum phase hypersonic vehicle model," IEEE Transactions on Systems, Man, and Cybernetics: Systems, vol. 51, no. 2, pp. 1107-1115, 2019.

[26] J.-J. E. Slotine, W. Li et al., Applied Nonlinear Control, Prentice-Hall, Englewood Cliffs, NJ, USA, 1991.

[27] L. Yao and W. J. Pan, "Fuzzy adaptive controller with modulated membership function for a mimo uncertain nonlinear system," in Proceedings of the Fourth International Conference on Innovative Computing, Kaohsiung, Taiwan, December 2009. 